\title{
Does Global Economic Uncertainty Matter for the Volatility and Hedging Effectiveness of Bitcoin?*
}

\author{
Libing Fang \\ School of Management and Engineering, Nanjing University, Nanjing, Jiangsu, \\ China. \\ Email: lbfang@nju.edu.cn \\ Elie Bouri ${ }^{\#}$ \\ USEK Business School, Holy Spirit University of Kaslik, Jounieh, Lebanon. \\ Email: eliebouri@usek.edu.lb \\ Rangan Gupta \\ Department of Economics, University of Pretoria, Pretoria, 0002, South Africa. \\ Email: rangan.gupta@up.ac.za \\ David Roubaud \\ Montpellier Business School, Montpellier, France. \\ Email: d.roubaud@montpellier-bs.com
}

\begin{abstract}
We assess whether the long-run volatilities of Bitcoin, global equities, commodities, and bonds are affected by global economic policy uncertainty. Empirical results provide evidence supporting that, except for the case of bonds. We further examine whether the correlation between Bitcoin and global equities, commodities, and bonds are affected by global economic policy uncertainty and the results reveal that global economic policy uncertainty has a negative significant impact on the Bitcoin-bonds correlation, and a positive impact on both Bitcoin-equities and Bitcoin-commodities correlations, suggesting a possibility for Bitcoin to act as a hedge under specific

\footnotetext{
* The authors would like to thank Professor Honghai Yu for many helpful comments, and Sifan Ding for excellent research assistance.

${ }^{*}$ Corresponding author.
} 
economic uncertainty conditions. Interestingly, the hedging effectiveness of Bitcoin for both global equities and global bonds enhances slightly after considering the level of global economic policy uncertainty. Implications for investors and policy-makers are discussed.

Keywords: Hedging effectiveness; Bitcoin; equities; commodities; bonds

JEL Codes: C10; G11.

\section{Introduction}

Proposed as an alternative to the fiat currencies, Bitcoin is a decentralized digital currency independent from sovereign governments, centralized institutions, and banking systems, and is often seen as a remedy to the ineffectiveness of standard economic and financial structures (Demir et al., 2018). In times of economic unrest and weak trust, the appeal of Bitcoin among investors and practitioners increases, making Bitcoin to shine (Bouri et al., 2017b; Luther and Salter, 2017; Demir et al., 2018).

Many methods have been used to study the relationship between Bitcoin and conventional assets, such as vector autoregressive models (Ciaian et al., 2016), regression analysis (Bouri et al., 2017; Baur et al., 2018), autoregressive distributed lag (Ciaian et al., 2016), wavelet coherence (Kristoufek, 2015), unconditional connectedness in the time-frequency domain (Corbet et al., 2018b), directed acyclic graph approach (Ji et al., 2018), univariate GARCH models (Dyhrberg, 2016), and multivariate GARCH models (Bouri et al., 2017; Guesmi et al., 2018). Most of those studies indicate that Bitcoin is very weakly correlated with conventional assets such 
as equities, bonds, and commodities (e.g., Bouri et al., 2017a; Baur et al., 2018; Corbet et al., 2018b; Guesmi et al., 2018; Ji et al., 2018), suggesting significant benefits for portfolio diversification and risk management (Brière et al., 2015; Bouri et al., 2017; Guesmi et al., 2018). In fact, since Bitcoin seems to behave independently ${ }^{1}$ from economic and financial developments (e.g., Kristoufek, 2015; Polasik et al., 2015), it can act as a hedge (Bouri et al., 2017a; Guesmi et al., 2018). Interestingly, Ciaian et al. (2016) find a significant role for macro-financial development (measured by the Dow Jones Index, exchange rate and crude oil price) in driving Bitcoin price in the short-run, while that role is insignificant in in long-run. Recently, Demir et al. (2018) indicate that Economic Policy Uncertainty (EPU) can be used to predict Bitcoin returns ${ }^{2}$, arguing that an increase in economic policy uncertainties makes the trust of investors in the global financial system and conventional currencies to decrease, and subsequently the attractiveness of Bitcoin to increase. Demir et al. (2018) suggest the need for policy-makers and investors to have a close eye on the effect of uncertainties in economic policies on Bitcoin returns.

While the above discussion shows that the relationship between Bitcoin and each of EPU, stocks, bonds, and commodities are somewhat explored, the related literature lacks a clear understanding of the influences determining the short-run and long-run relationships between Bitcoin and other financial assets contingent on a measure of macroeconomic uncertainty, such as EPU.

This paper complements the above lines of research by examining whether EPU affects the correlation between Bitcoin and conventional assets using a different model, called the DCC-MIDAS approach (Colacito et al., 2011). The idea behind DCC-MIDAS is to (1) decompose the Bitcoin-conventional assets correlation into

\footnotetext{
${ }^{1}$ Compared to conventional assets, Bitcoin is exposed and determined by a unique set of non-financial and non-economic variables that include social sentiment (Kristoufek, 2015; Bouoiyour and Selmi, 2015; Polasik et al., 2015), energy prices (Hayes, 2017), user anonymity (Ober et al., 2013), computer programming enthusiasts (Yelowitz and Wilson, 2015).

${ }^{2}$ Demir et al. (2018) build on the work of Bouri et al. (2017b) that showed the ability of Bitcoin to hedge global uncertainty, as measured by the first principal component of the implied volatility indices of 14 developed and developing stock markets.
} 
long- and short-run components and (2) combine daily Bitcoin and financial assets returns with a monthly EPU index ${ }^{3}$. By allowing both long- and short-run correlations to be affected by EPU, we can also make inferences on the (out-of-sample) hedging effectiveness of Bitcoin against conventional assets depending on the effect of EPU on those correlations. Such an examination would add to the standard literature that generally makes inferences on the hedging ability of Bitcoin by measuring directly the correlation between Bitcoin and conventional assets (Bouri et al., 2017a; Baur et al., 2018; Corbet et al., 2018b; Guesmi et al., 2018; Ji et al., 2018). Furthermore, the examination would provide useful implications to investors and practitioners, especially in assessing the hedging effectiveness of Bitcoin against conventional assets depending on the effect of EPU and in improving the efficiency of asset allocation between Bitcoin and financial assets depending on the effect of EPU. This is important because a more accurate estimation of relationships between Bitcoin and financial assets depending on the level of EPU is crucial for the construction of a diversified portfolios and in managing risk. For example, periods of negative correlation (uncorrelation) between the prices of Bitcoin and financial assets offer an opportunity for optimizing portfolio and hedging strategies depending on whether the level of EPU is high, medium, or low. In fact, the literature generally argues that an increase in EPU may positively influence commodities (Shahzad et al., 2017; Yin and Han, 2014), bonds (Ioannidis and Ka, 2018), and Bitcoin (Demir et al., 2018), as these three assets possess some hedging properties.

The positive effect of EPU on Bitcoin-commodities (Bitcoin-bonds) correlation implies that Bitcoin will serve as a hedge when the level of EPU is low and when the Bitcoin-commodities (Bitcoin-bonds) correlation is negative. However, when the level of EPU is high, Bitcoin might not serve as a good hedge. As for the negative effect of EPU on Bitcoin-stock correlation and given an increase in EPU can

\footnotetext{
${ }^{3}$ As argued by Colacito et al. (2011), component models - such as the DCC-MIDAS - are not only used widely in modelling volatility dynamics, but their component structures allow for a parsimonious representation of complex dependence structures. Importantly, their components are generally linked to macroeconomic sources.
} 
adversely affect stock (Arouri et al., 2016), Bitcoin will serve as a hedge when the level of EPU is high and when the Bitcoin-gold correlation is negative. However, when the level of EPU is low, Bitcoin may not serve as a good hedge.

Our main results indicate that the long-term volatility of Bitcoin, MSCI, and S\&P GSCI are significantly affected by EPU, although the effect on the long-run volatility of Bitcoin is different as compared to MCSI and PIMCO. Furthermore, GEPU has a negative significant impact on the Bitcoin-PIMCO correlation, and a positive impact on both Bitcoin-MSCI and Bitcoin-GSCI correlations, suggesting a possibility for Bitcoin to act as a hedge. However, the hedging effectiveness of Bitcoin increased slightly after incorporating EPU (for both MSCI and PIMCO).

The rest of the paper continues as follows. Section 2 provides a review of the literature on the hedging ability of Bitcoin against conventional assets, especially stocks. Section 3 describes the data and methods. Section 4 presents and discusses the empirical results. Finally, section 5 concludes.

\section{Related studies}

Since its launch in 2009 as the first digital currency, Bitcoin has substantially grown in popularity because of its independence from governments and other central authorities. In fact, Bitcoin technology relies on mass collaboration via its genuine decentralized network through which Bitcoin transactions are validated and settled to prevent fraud. The integrity of the transactions is maintained by a distributed ledger, called blockchain, where all transactions are stored digitally.

The debate on Bitcoin continues to ascent with some evidence on its speculative nature (Cheah and Fry, 2015; Baur et al., 2018), bubble formation (Cheah and Fry, 2015; Bouri et al., 2018; Corbet et al. 2018a), high price volatility (Molnár et al., 2015), mounting regulatory scrutiny and market manipulation (Gandal et al., 2018), cyber-attacks, and exchange security flaws. Some other studies try to raise awareness

\footnotetext{
${ }^{4}$ https://hackernoon.com/breaking-news-bitcoin-market-manipulation-detected-by-artificial-intelligence -a4534b7be369
} 
among practitioners of the possibility for Bitcoin to be part of money laundering (Moser et al., 2013) or even Ponzi schemes (Vasek and Moore, 2015). While other studies consider Bit coin to be a medium of exchange but weakly related to global macroeconomic aggregates (e.g., Polasik et al., 2015). Interestingly, the meteoric price appreciation of Bitcoin made its total market value to reach more than \$216 billion at the end of December 2017 (https://coinmarketcap.com/).

Importantly, a stream of research that continues to attract the attention of scholars and experts is the ability of Bitcoin to diversify/hedge the downside risk of financial assets. Using univariate GRACH models, Dyhrberg (2016) shows that Bitcoin can serve as a hedge for both the FTSE 100 and the US dollar. Bouri et al. (2017a) use a DCC-GRACH model and a regression analysis based on quantile dummies to indicate that Bitcoin can diversify extreme down movements in equity returns. Using a quite similar method, Baur et al. (2018) show evidence that Bitcoin is weakly correlated with equities suggesting a diversification ability in both in normal and stress periods, and that despite claims by the authors that Bitcoin is "mainly used as a speculative investment and not as an alternative currency and medium of exchange”. Using a multivariate GARCH model, Guesmi et al. (2018) show that Bitcoin has a hedging potential for different financial assets, including equities. Similar results were reported by Ji et al. (2018) who use a graph theory approach and variance decomposition measures. Corbet et al. (2018b) use connectedness measures in the time and frequency domains and provide evidence similar to that found in the above-mentioned studies, i.e., Bitcoin is mostly independent from the global financial system and conventional assets and thus can offer diversification benefits. However, Klein et al. (2018) apply a combination of methods and find that the conditional variance properties of Bitcoin are different from those of Gold. In particular, Bitcoin appears to move in tandem with downward stock markets. A portfolio analysis show that Bitcoin has unstable hedging capabilities.

Scholars often look at the direct correlation between Bitcoin and conventional assets such as stocks, commodities, and bonds and report evidence of very weak 
correlation (Bouri et al., 2017a; Baur et al., 2018; Corbet et al., 2018b; Guesmi et al., 2018; Ji et al., 2018). Bitcoin is mostly isolated from economic and financial variables, probably due to the unique factors that determine its prices such as social sentiment (Kristoufek, 2015; Bouoiyour and Selmi, 2015), ratio of exchange-trade volume and the hash rate (Bouoiyour and Selmi, 2015), energy prices (Hayes, 2017), user anonymity (Ober et al., 2013), computer programming enthusiasts (Yelowitz and Wilson, 2015), technology (Li and Wang, 2017), cyber-attacks (Böhme et al., 2015), increased stock market uncertainty (Bouri et al., 2017b), and economic policy uncertainty (Demir et al., 2018).

However, there is still a lack of understanding of the long-run and short-run relationships between Bitcoin and financial assets related to macroeconomic variables such as EPU. In a related point, it is unclear whether the hedging effectiveness of Bitcoin is affected by the state of EPU. To address these issues, GARCH-MIDAS (Engle et al., 2013) and its extension the DCC-MIDAS (Colacito et al., 2011) appear as suitable modelling frameworks. These components models have been widely used in the finance literature. They attracted the interest of scholars due to their ability to decompose the correlation between assets into long- and short-run components, which are generally linked to macroeconomic sources, and combine data from different levels of frequencies.

\section{Data and methods}

\subsection{Data}

This study uses daily and weekly data. Daily data are expressed in USD and include the closing levels of Bitcoin, global equities, commodities, and bonds, measured respectively by the CoinDesk Bitcoin Price Index, the MSCI world stock index, the S\&P GSCI Commodity index, and the PIMCO Investment-Grade Corporate Bonds index (Ji et al., 2018). Data for Bitcoin price are collected from www.coindesk.com/price, whereas the rest of data are extracted from DataStream. Regarding uncertainty data, we use the monthly index of global economic policy 
uncertainty (GEPU), which is based on the work of Baker et al., (2016), and collected from http://www.policyuncertainty.com/global_monthly.html. ${ }^{\underline{5}}$ The GEPU Index is a (PPP-adjusted) GDP-weighted average of national EPU indices for 20 countries: Australia, Brazil, Canada, Chile, China, France, Germany, Greece, India, Ireland, Italy, Japan, Mexico, the Netherlands, Russia, South Korea, Spain, Sweden, the United Kingdom, and the United States. Note that, each national EPU index reflects the relative frequency of own-country newspaper articles that contain a trio of terms pertaining to the economy (E), policy $(\mathrm{P})$ and uncertainty $(\mathrm{U})$.

The sample period starts at 21 September 2010, as depicted by the availability of the PIMCO index, and ends at 26 January 2018. The empirical analyses are conducted with the log-return series, barring the GEPU, which is in its raw level-form given that it is stationary. Table A1 in the Appendix provides the summary statistics. Bitcoin has the highest mean and standard deviation. MSCI World, S\&P GSCI, and PIMCO are negatively skewed. All return series, along with the GEPU, are non-normally distributed as indicated by the Jarque-Bera statistics.

\subsection{GARCH-MIDAS model}

To directly investigate how long-run bitcoin, MSCI, S\&P GSCI, and PIMCO index volatility evolves along with the GEPU (Global Economic Policy Uncertainty), we rely on the GARCH-MIDAS model proposed by Engle et al. (2013). In our approach, the long-term volatility is modeled on the basis of the lagged GEPU and a MIDAS polynomial that applies to monthly GEPU. The GARCH-MIDAS model is specified as follows:

$$
r_{i, t}=\mu_{i}+\varepsilon_{i, t}=\mu_{i}+\sqrt{m_{i, \tau} \cdot g_{i, t}} \xi_{i, t}
$$

where $r_{t}$ is a bivariate vector of returns, in which $r_{i, t}$ denotes the return of Bitcoin,

\footnotetext{
${ }^{5}$ We opted for the GEPU index instead of EPU indices of particular countries or regions because the use of Bitcoin as a global digital currency necessitates the choice of a global measure of economic uncertainty such as the GEPU index.
} 
MSCI, S\&P GSCI, and PIMCO index on day $t ; g_{i, t}$ is the short-run variance component on day $t$ accounting for daily fluctuations that are assumed to be short-lived; while $m_{i, \tau}$ is defined as the long-run component that varies with monthly frequency $\tau ; N_{t}$ denotes the number of days in a month. The short-run component $g_{i, t}$ follows a GARCH $(1,1)$ process,

$$
g_{i, t}=\left(1-\alpha_{i}-\beta_{i}\right)+\alpha_{i} \frac{\left(r_{i, t-1}-\mu_{i}\right)^{2}}{m_{i, \tau}}+\beta_{i} g_{i, t-1}
$$

with $\alpha_{i}>0$ and $\beta_{i}>0, \alpha_{i}+\beta_{i}<1$.

by smoothing realized volatility (RV) in the spirit of the MIDAS regression and MIDAS filtering, we specify the $m_{i, \tau}$ component as follows:

$$
\begin{aligned}
& \log \left(m_{i, \tau}\right)=m_{i}+L_{i} \\
& L_{i}=\theta_{i} \sum_{k=1}^{K} \varphi_{k}\left(\omega_{1, i}, \omega_{2, i}\right) R V_{\tau-k} \\
& R V_{\tau}=\sum_{t=1}^{N_{t}} r_{i, t}^{2}
\end{aligned}
$$

where $K$ is the number of periods over which we smooth the volatility.

To study the impact of GEPU on the return variance, we extend Equation (3) by introducing GEPU and RV,

$$
\begin{aligned}
& \log \left(m_{i, \tau}\right)=m_{i}+L_{i, 1}+L_{i, 2} \\
& L_{i, 1}=\theta_{i, R V} \sum_{k=1}^{K} \varphi_{k}\left(\omega_{i, 1}^{R V}, \omega_{i, 2}^{R V}\right) R V_{\tau-k} \\
& L_{i, 2}=\theta_{i, \mathrm{X}} \sum_{k=1}^{K} \varphi_{k}\left(\omega_{i, 1}^{G E P U}, \omega_{i, 2}^{G E P U}\right) X_{\tau-k}^{G E P U}
\end{aligned}
$$

where $\mathrm{X}_{\tau-k}^{G E P U}$ represents the change in GEPU, defined as the difference of logarithms of GEPU. The weighting scheme in specification (4) comprises the so-called beta weights defined by

$$
\varphi_{k}\left(w_{i}\right)=\frac{\left(1-\frac{k}{K}\right)^{w_{i}-1}}{\sum_{s=1}^{K}\left(1-\frac{s}{K}\right)^{w_{i}-1}}, \forall k=1, \ldots, K
$$


Equations (1) to (5) form the GARCH-MIDAS model of time-varying conditional variance with fixed time-span RVs and the parameter space $\Theta=\left\{\varepsilon_{i}, \alpha_{i}, \beta_{i}, m_{i}, \theta_{i}, \omega_{i, 1}, \omega_{i, 2}\right\}$.

Finally, the total conditional variance is defined as:

$$
\sigma_{i, t}^{2}=m_{i, \tau} \cdot g_{i, t}
$$

\subsection{Estimation of models}

As shown in Equations 3-4, and instead of using a single model specification, as in Asgharian et al. (2013), we consider two different model specifications (GARCH-MIDAS with RV and GARCH-MIDAS with RV+GEPU). In both specifications, the short-term variance $g_{i, t}$ remains the same, while the definition of the long-term variance component $m_{i, \tau}$ differs.

Equation 3 represents the benchmark MIDAS model, where $R V$ is added to the long-term component of the variance. This benchmark specification, named GARCH-MIDAS with RV, uses the monthly RV as an explanatory variable.

Equation 4 extends the GARCH-MIDAS model with the aim of investigating whether the GEPU variable has an additional explanatory power over RV and thus capturing the potential information explained by both GEPU and RV. Accordingly, we modify the long-term component by simultaneously including both $R V$ and a macro variable $\mathrm{X}_{\tau-k}^{G E P U}$ as explanatory variables. We refer to this model as GARCH-MIDAS with RV+GEPU ${ }^{6}$.

\subsection{DCC-MIDAS model}

The DCC-MIDAS model of Colacito et al. (2011) naturally extends the GARCH-MIDAS model to dynamic correlations. And slightly different from Conrad

\footnotetext{
${ }^{6}$ Consistent with our findings, we set $\omega_{1}^{R V}=1$ here, and estimate the weighting schemes for RV and GEPU.
} 
et al. (2014), we extend the specification by allowing the long-run correlation to depend directly on the lagged GEPU. In this paper, the conditional correlation between Bitcoin and the other indices (MSCI, GSCI and PIMCO) is given as $R_{t}=\operatorname{diag}\left(Q_{t}\right)^{-1 / 2} Q_{t} \operatorname{diag}\left(Q_{t}\right)^{-1 / 2}$, where $Q_{t}$ is the short-run correlation component. We define $q_{i j, t}$ as the elements in $Q_{t}$ as:

$$
q_{i j, t}=(1-\mathrm{a}-\mathrm{b}) \bar{\rho}_{i j, \tau}+a \xi_{i, t-1} \xi_{j, t-1}+b q_{i j, t-1}
$$

where $\xi_{i, t-1}$ and $\xi_{j, t-1}$ are the standardized residuals from GARCH-MIDAS models; $\bar{\rho}_{i j, \tau}$ is a slowly moving long-run correlation defined by a Fisher-z transformation of the correlation coefficient as follows:

$$
\bar{\rho}_{i j, \tau}=\frac{\exp \left(2 z_{i j, \tau}\right)-1}{\exp \left(2 z_{i j, \tau}\right)+1}
$$

$\bar{\rho}_{i j, \tau}$ remains locally constant during a long-term $\tau$ period. While $z_{i j, \tau}$ is depended directly on the lagged GEPU:

$$
Z_{i j, \tau}=m_{c}+\theta_{c} \sum_{k=1}^{K_{c}} \delta_{k}\left(w_{c}\right) \mathrm{GEPU}_{t-k}
$$

The correlation $\rho_{t}$ is obtained by the specification: $\rho_{t}=\frac{q_{12, t}}{\sqrt{q_{11, t} q_{22, t}}}$, which is the correlation between the standardized residuals. The weighting scheme $\delta_{k}\left(\mathrm{w}_{c}\right)$ is defined similarly to $\varphi_{k}\left(w_{v}^{i}\right)$, and the parameter $\theta_{c}$ denotes the effect of the sentiment on the long-term correlation.

\subsection{Hedging Performance}

Now, we consider whether the incorporation of GEPU improve the hedging performance of Bitcoin with the three indices (MSCI, GSCI and PIMCO). Specifically, we test for the out-of-sample hedging performance of different strategies and compare between them. The return on a hedge portfolio is denoted by:

$$
R_{H, t}=R_{B, t}-\gamma_{t} R_{I, t}
$$

where $R_{B, t}$ is the return on the Bitcoin position; $R_{I, t}$ is the return on the three 
indices position, and $\gamma_{t}$ is the hedge ration. The variance of the hedge portfolio conditional on the information set at time $\mathrm{t}-1$ is:

$$
\operatorname{var}\left(R_{H, t} \mid I_{t-1}\right)=\operatorname{var}\left(R_{B, t} \mid I_{t-1}\right)-2 \gamma_{t} \operatorname{cov}\left(R_{B, t}, R_{I, t} \mid I_{t-1}\right)+\gamma_{t}^{2} \operatorname{var}\left(R_{I, t} \mid I_{t-1}\right)
$$

In subsection 3.3, we obtain conditional covariance matrix $\mathbf{H}_{t}$ from DCC-MIDAS. Due to the assumption of two assets, $\mathbf{H}_{t}$ is a $2 \times 2$ symmetric matrix. Thus, $\operatorname{var}\left(R_{B, t} \mid I_{t-1}\right)$ and $\operatorname{var}\left(R_{I, t} \mid I_{t-1}\right)$ are diagonal elements, and $\operatorname{cov}\left(R_{B, t}, R_{I, t} \mid I_{t-1}\right)$ is the non-diagonal element.

The optimal hedge ratios (OHRs) are defined as the value of $\gamma_{t}$ which minimizes the conditional variance of the hedge portfolio, as follows:

$$
\gamma_{t}^{*} \mid I_{t-1}=\frac{\operatorname{cov}\left(R_{B, t}, R_{I, t} \mid I_{t-1}\right)}{\operatorname{var}\left(R_{I, t} \mid I_{t-1}\right)}
$$

For comparing the hedging performances of different models, we use the following criteria. An effective hedging strategy should have a noticeable impact on the risk (standard deviation) without a noticeable impact on the return (mean) when included in a portfolio. The measure is hedging effectiveness (HE) index which defined as:

$$
H E=\frac{\operatorname{var}_{\text {unhedged }}-\operatorname{var}_{\text {hedged }}}{\operatorname{var}_{\text {unhedged }}}
$$

where $\operatorname{var}_{\text {hedged }}$ is the variance of the hedge portfolio return $R_{H, t}$ and $\operatorname{var}_{\text {unhedged }}$ is the variance of the unhedged portfolio return $R_{B, t}$. A higher HE indicates a higher hedging effectiveness and larger risk reduction, so a hedging strategy with a higher HE is regarded as a superior hedging strategy.

\section{Empirical results}

In subsection 4.1, we first analyze GARCH-MIDAS model to describe the long-run volatilities of Bitcoin, MSCI, S\&P GSCI and PIMCO based on RV and 
subsequently extend the model by allowing the long-term volatility to be affected by GEPU. Next, we present in subsection 4.2 the estimation results of the DCC-MIDAS specification that focuses on the long-run correlation between Bitcoin and the three indices (MSCI, S\&P GSCI and PIMCO). Lastly, we compute in subsection 4.3 the hedging ratio to examine whether incorporating GEPU could improve the hedging effectiveness of Bitcoin.

\subsection{Impact of GEPU on long-run volatility}

Table 1 presents the estimated results from the GARCH-MIDAS model that was used to describe the long-run volatilities of Bitcoin, MSCI, S\&P GSCI and PIMCO based on the RV. Most of the parameters in the equations for returns and the short-term variance component are significant at the $1 \%$ level, indicating a clustering pattern in the short-term return variance. The sums of $\alpha$ and $\beta$ are noticeably close to 1 , confirming the strong volatility persistence effect. We also note that the estimated parameter $\theta_{v}$ is significantly positive for MCSI, implying that long-term volatility of world equities increases with the RV. However, $\theta_{v}$ is negative and significant for the Bitcoin and commodity markets. The $\theta_{v}$ of Bitcoin has a much bigger magnitude than that of the other indices, which indicates that RV has a multiplied effect on its long-run volatility.

Table 1. The GARCH-MIDAS model parameter estimates of RV (lags=3)

\begin{tabular}{ccccc}
\hline & Bitcoin & MCSI & GSCI & PIMCO \\
\hline$\mu$ & $0.0030^{* * *}$ & $0.0556^{* * *}$ & $-0.0438^{* *}$ & 0.0064 \\
& $(3.1561)$ & $(4.0715)$ & $(-2.4168)$ & $(0.9323)$ \\
$\alpha$ & $0.1051^{* * *}$ & $0.1557^{* * *}$ & $0.0873^{* * *}$ & $0.0734^{* * *}$ \\
& $(26.1510)$ & $(10.0035)$ & $(11.2774)$ & $(11.8911)$ \\
$\beta$ & $0.8938^{* * *}$ & $0.7942^{* * *}$ & $0.9127^{* * *}$ & $0.9041^{* * *}$ \\
\multirow{3}{*}{$\theta_{v}$} & $(221.6454)$ & $(38.4214)$ & $(137.7402)$ & $(58.5870)$ \\
& $-3.3490^{* * *}$ & $0.0255^{* * *}$ & $-0.0119^{* * *}$ & -0.0305 \\
& $(-7.8421)$ & $(4.5021)$ & $(-3.0957)$ & $(-0.5458)$ \\
\hline & $2.2626^{* * * *}$ & $1.0010^{* * *}$ & $2.9744^{* *}$ & 25.5195 \\
\hline
\end{tabular}




\begin{tabular}{lllll}
\hline & $(4.8713)$ & $(71.6072)$ & $(2.5531)$ & $(0.0000)$ \\
\multirow{2}{*}{$m_{v}$} & $-3.2784^{* * *}$ & $-0.8571^{* * *}$ & $-0.8498^{*}$ & $-2.0681^{* * *}$ \\
& $(-13.3916)$ & $(-6.4199)$ & $(-1.7337)$ & $(-8.9336)$ \\
\hline
\end{tabular}

Notes: The table reports the estimates of the GARCH-MIDAS coefficients for bitcoin and MSCI, S\&P GSCI, and PIMCO index. The sample covers 21/09/2010 to 31/12/2017. ***, **, and * indicate significance at the $1 \%$, $5 \%$, and $10 \%$ levels.

Table 2 presents the estimated results from the extended GARCH-MIDAS specification, through which the long-run volatility was allowed be affected by both RV and GEPU. Results indicate that, after incorporating GEPU in the MIDAS equation, the coefficients $\theta_{R V}$ are still significantly positive for MCSI while significantly negative for the Bitcoin and S\&P GSCI. The estimated parameters $\theta_{X}$, which indicate the response of long-run volatility to GEPU, are all significant at least at 5\% level. Positive $\theta_{X}$ for Bitcoin and S\&P GSCI indicates that GEPU has a positive influence on the long-run volatility of Bitcoin and commodity markets. By contrast, negative $\theta_{X}$ for MCSI and PIMCO indicates that the influence of the GEPU on stock and bond markets is the opposite. Those results preliminarily show that GEPU has different impact on the Bitcoin and the other indices, which necessitates further investigation on the correlation and hedging performance, as shown in the following subsections.

Table 2. The GARCH-MIDAS model parameter estimates of RV and GEPU (lags=3)

\begin{tabular}{ccccc}
\hline & Bitcoin & MCSI & GSCI & PIMCO \\
\hline$\mu$ & $0.0030^{* * *}$ & $0.0554^{* * *}$ & $-0.1183^{* * *}$ & 0.0075 \\
& $(3.1769)$ & $(4.0458)$ & $(-8.1882)$ & $(1.1099)$ \\
$\alpha$ & $0.1063^{* * *}$ & $0.1577^{* * *}$ & $0.0993^{* * *}$ & $0.0534^{* * *}$ \\
& $(24.6314)$ & $(10.2419)$ & $(11.0191)$ & $(6.9092)$ \\
\multirow{2}{*}{$\beta$} & $0.8926^{* * *}$ & $0.7906^{* * *}$ & $0.9007^{* * *}$ & $0.9335^{* * *}$ \\
& $(207.5208)$ & $(37.2451)$ & $(116.8184)$ & $(76.3695)$ \\
$\theta_{R V}$ & $-3.0813^{* * *}$ & $0.0282^{* * *}$ & $-0.0086^{* *}$ & -0.0559 \\
\multirow{2}{*}{$\theta_{X}$} & $(-6.2092)$ & $(4.3817)$ & $(-1.9660)$ & $(-0.8424)$ \\
& $0.9332^{* *}$ & $-1.7652^{* *}$ & $4.2657^{* * *}$ & $-1.1210^{* * *}$ \\
$\omega_{R V}$ & $(2.3313)$ & $(-2.5499)$ & $(12.9470)$ & $(-4.4666)$ \\
& $2.1671^{* * *}$ & $1.0010^{* * *}$ & 6.6317 & $1.0010^{* * *}$ \\
\hline
\end{tabular}




\begin{tabular}{lllll}
\hline$\omega_{X}$ & $1.0532^{* * *}$ & $1.0010^{* * *}$ & $5.7318^{* * *}$ & 25.6331 \\
& $(18.2814)$ & $(66.1734)$ & $(3.0746)$ & $(0.0000)$ \\
$m_{v}$ & $-3.3377^{* * *}$ & $-0.8950^{* * *}$ & $-3.0668^{* * *}$ & $-2.0242^{* * *}$ \\
& $(-12.6437)$ & $(-6.3559)$ & $(-3.3702)$ & $(-7.5760)$ \\
\hline
\end{tabular}

Notes: The table reports the estimates of the GARCH-MIDAS coefficients for bitcoin and MSCI, S\&P GSCI, and PIMCO index. The sample covers 21/09/2010 to 31/12/2017. ***,**, and * indicate significance at the $1 \%$, $5 \%$, and $10 \%$ levels.

\subsection{Long term correlation between Bitcoin and the other indices}

We first examine the long-term correlation based on the realized correlation (RC), without adding exogenous variables (i.e. RV and GEPU). The Columns 1-3 in Table 3 show the DCC-MIDAS dynamic model parameter estimates of Bitcoin and the other indices (MSCI, S\&P GSCI and PIMCO). $m_{v}$ reflects how the Bitcoin- $\mathrm{X}^{7}$ correlation responds to RC shocks. We find a statistically significant positive $m_{v}$ in Column 1 , suggesting that the impact of RC is specified. As for the impact of RC in the other cases (Columns 2 and 3), it is insignificant. Then, we subsequently estimate the DCCMIDAS-RV-GEPU parameters by incorporating monthly RV and GEPU into long-term correlation.

Table 3. The DCC-MIDAS model with parameter estimates of Bitcoin (lags=3)

\begin{tabular}{|c|c|c|c|c|c|c|}
\hline & $\mathrm{RC}+\mathrm{MCSI}$ & $\mathrm{RC}+\mathrm{GSCI}$ & $\begin{array}{l}\text { RC+PIMC } \\
\mathrm{O}\end{array}$ & $\begin{array}{l}\text { RC+MSCI+ } \\
\text { GEPU }\end{array}$ & $\begin{array}{l}\text { RC+GSCI+ } \\
\text { GEPU }\end{array}$ & $\begin{array}{l}\text { RC+PIMCO+ } \\
\text { GEPU }\end{array}$ \\
\hline$\alpha$ & $\begin{array}{l}0.0000 \\
(0.0068)\end{array}$ & $\begin{array}{l}0.0000 \\
(0.0010)\end{array}$ & $\begin{array}{l}0.0182 * * \\
(2.2231)\end{array}$ & $\begin{array}{l}0.0000 \\
(0.0000)\end{array}$ & $\begin{array}{l}0.0000 \\
(0.0000)\end{array}$ & $\begin{array}{l}0.0000 \\
(0.0053)\end{array}$ \\
\hline$\beta$ & $\begin{array}{l}1.0000 * * * \\
(976.3323)\end{array}$ & $\begin{array}{l}1.0000 * * * \\
(1000.6134)\end{array}$ & $\begin{array}{l}0.9818 * * * \\
(96.4017)\end{array}$ & $\begin{array}{l}1.0000 * * * \\
(69.7975)\end{array}$ & $\begin{array}{l}0.9985 * * * \\
(437.9623)\end{array}$ & $\begin{array}{l}1.0000 * * * \\
(646.8217)\end{array}$ \\
\hline$m_{v}$ & $\begin{array}{l}3.5888 * * * \\
(30.7710)\end{array}$ & $\begin{array}{l}4.0454 \\
(0.8744)\end{array}$ & $\begin{array}{l}4.3249 \\
(0.2555)\end{array}$ & $\begin{array}{l}-3.8847 * * * \\
(-59.4770)\end{array}$ & $\begin{array}{l}-0.1794 * * \\
(-2.3246)\end{array}$ & $\begin{array}{l}0.0141 \\
(0.1280)\end{array}$ \\
\hline$\theta_{R V}$ & & & & $\begin{array}{l}0.4249 * * * \\
(23.7371)\end{array}$ & $\begin{array}{l}-2.5908 * * \\
(-2.1156)\end{array}$ & $\begin{array}{l}0.9629 * * * \\
(18.7693)\end{array}$ \\
\hline$\theta_{\mathrm{x}}$ & & & & $\begin{array}{l}1.4552 * * * \\
(6.1325)\end{array}$ & $\begin{array}{l}7.4132 * \\
(1.9477)\end{array}$ & $\begin{array}{l}-0.8891 * * * \\
(-5.8333)\end{array}$ \\
\hline$\therefore$ & & & & $5.6371^{* * *}$ & 25.3401 & $5.0016 * * *$ \\
\hline
\end{tabular}

\footnotetext{
${ }^{7} \mathrm{X}$ denotes each of the other three indices (i.e., MSCI, GSCI and PIMCO).
} 


\begin{tabular}{llll} 
& $(5.4603)$ & $(0.2362)$ & $(2.8772)$ \\
$\omega_{\mathrm{x}}$ & $5.6371 * * *$ & $1.0010 * * *$ & $5.0020 * * *$ \\
& $(34.7774)$ & $(22.4556)$ & $\mathbf{( 5 1 . 1 2 1 4 )}$ \\
\hline
\end{tabular}

Notes: The table reports the estimates of the DCC-MIDAS coefficients for bitcoin and MSCI, S\&P GSCI, and PIMCO index. The sample covers $21 / 09 / 2010$ to 31/12/2017. ***, **, and * indicate significance at the $1 \%, 5 \%$, and $10 \%$ levels.

As shown in Columns 4-6 of Table 3, the results of $\theta_{R V}$ and $\theta_{X}$ are almost statistically significant, suggesting that the response of long-term correlation to RV and GEPU is intense. For the parameter estimates of $\theta_{X}$, it is significantly positive for the correlation of Bitcoin-PIMCO, but negative for the others. Accordingly, the GEPU has a rather ambiguous negative impact on the correlation of Bitcoin-PIMCO, and positive effect on the Bitcoin-MSCI and Bitcoin-GSCI. The negative effect of EPU on Bitcoin-PIMCO correlation suggests that investors can consider Bitcoin as a hedging asset for the PIMCO index during periods of high GEPU and negative Bitcoin-PIMCO correlation. On the contrary, the positive effect of GEPU on Bitcoin-MSCI and Bitcoin-GSCI correlations suggests that investors can treat Bit coin as a hedging asset for MSCI and S\&P GSCI indices during periods of low GEPU and negative Bitcoin-MSCI (Bitcoin-S\&P GSCI) correlation. Based on the above analysis, we examine in the following subsection whether GEPU can improve the hedging results of Bitcoin-X.

\subsection{Hedging performance}

We study whether the addition of GEPU can improve the hedging performance of Bitcoin. To this end, we substitute the covariance matrix, calculated by the DCC-MIDAS and DCC-MIDAS-GEPU, into equation (8), and then calculate the optimal hedge ratio. Results from Table 4 show that the hedging performance ${ }^{8}$ of Bitcoin increased after incorporating GEPU (for both MSCI and PIMCO), but its effect is less than $1 \%$.

\footnotetext{
${ }^{8}$ We use HE as a proxy for hedging performance.
} 
Table 4. Comparisons of the hedging performance of different indexes (lags=3).

\begin{tabular}{llll}
\hline & MCSI & GSCI & PIMCO \\
\hline HE & 0.0000 & 0.0000 & -0.0036 \\
\hline HE & MSCI+GEPU & GSCI+GEPU & PIMCO+GEPU \\
\hline
\end{tabular}

Notes: The table reports the estimates of hedging performance of bitcoin and MSCI, S\&P GSCI, and PIMCO index, with and without global economy uncertainty as macroeconomic proxy. The sample covers 21/09/2010 to $31 / 12 / 2017$. HE is a proxy for hedging performance.

It is clear that the incorporation of GEPU can slightly reduce the portfolio risk and improve the effectiveness of hedging of Bit coin for both MSCI and PIMCO, which is not the case for S\&P GSCI. This may be because the correlation between Bitcoin and commodity index is relatively low, and the hedging effect between the two assets is not substantial. In addition, the PICMO bond index has the best hedging improvement, probably because the correlation between Bitcoin and PICMO is relatively higher than that for the stock market and commodity index and because GEPU fits appropriately the dynamic correlation coefficient. Finally, the hedging performance for all the three indices (MSCI, S\&P GSCI, PIMCO) improves less than 1\%, We argue that Bitcoin, as digital currency, is considerably affected by network sentiment and is thus very different from the other assets under study. In light of the above and although GEPU can improve the correlation prediction, the correlation is still low, and the hedging effect is not considerable.

All in all, the above results seem to be affected by the high volatility of Bitcoin that is generally driven much more by the traders' attention and speculative sentiment compared to the global economic uncertainty. This finding partially contradicts with that of Demir et al. (2018). However, the findings also suggest that the volatility of global stock and bond indices are more linked to the state of global economic uncertainty rather than to the driving factors of Bitcoin. Therefore, the impact of GEPU on the hedging performance of Bitcoin was found to be small.

\section{Concluding remarks}

We extend the related literature on the hedging ability of Bitcoin against 
conventional assets by examining the impact of global economic policy uncertainty on the long-run correlation between Bitcoin and global equities, commodities, and bonds as well as on Bit coin hedging effectiveness.

Empirical results are summarized as follows. The application of GARCH-MIDAS models shows that the long-term volatility of Bitcoin, MSCI, and S\&P GSCI are significantly affected by EPU, although the effect on the long-run volatility of Bitcoin is different as compared to MCSI and PIMCO. The application of the DCC-MIDAS reveals that the GEPU has a negative significant impact on the Bitcoin-PIMCO correlation, and a positive impact on both Bitcoin-MSCI and Bitcoin-GSCI correlations, suggesting a possibility for Bitcoin to act as a hedge under specific economic uncertainty conditions. Further analysis shows that the hedging effectiveness of Bitcoin for both MSCI and PIMCO increased slightly after accounting for the impact of GEPU.

While the above findings add to that of Bouri et al. (2017b), Demir et al. (2018) and Guesmi et al. (2018), they have implications regarding investment decisions and policy making. The fact that Bitcoin volatility is affected by the state of economic uncertainty suggests that investors and practitioners in the Bitcoin market have to closely watch the level of the global economic policy uncertainty while making investment decision involving the volatility of Bitcoin, which represents a central input into options pricing. Therefore, investors can use information about the state of global economic uncertainty in enhancing the predictions of Bitcoin volatility. This might be useful when making risk management inferences that involve the potential launch of options contracts on Bitcoin and Bitcoin futures that are now traded on the CME Group and the CBOE. Regarding the finding on the weak effect of the state of the GEPU on the hedging ability of Bitcoin, it implies that investors cannot substantially enhance the hedging performance of Bitcoin under different economic uncertainty conditions. Regarding policy-makers, they can be concerned by our findings given mounting interest on the potential effect of contagion from the cryptocurrency market and its main element, Bitcoin, to the global financial system. 
This issue also concerns the case of central banks that seek to consider Bitcoin as part of their foreign reserves or test their own versions of digital currencies. Although our work highlights, so far, the weak role of global economic uncertainty in driving the long-run volatility of Bit coin, it would be wise to continue the monitoring of the Bitcoin market (European Central Bank, 2012).

Further research can consider country level data on equity and EPU indices, and/or the time-variability in the effect of EPU on the hedging ability of Bitcoin.

\section{References}

Arouri, M., Estay, C., Rault, C., Roubaud, D., 2016. Economic policy uncertainty and stock markets: Long-run evidence from the US. Finance Research Letters, 18, 136-141.

Asgharian, H., Hou, A. J., Javed, F., 2013. The importance of the macroeconomic variables in forecasting stock return variance: A GARCH-MIDAS approach. Journal of Forecasting, 32(7), 600-612.

Baker, S., N. Bloom, and S. Davis (2016). Measuring economic policy uncertainty. The Quarterly Journal of Economics, 131(4), 1593-1636.

Baur, D.G., Hong, K., Lee, A.D., 2018. Bitcoin: Medium of Exchange or Speculative Assets? Journal of International Financial Markets, Institutions and Money, 54, 177-189.

Böhme, R., Christin, N., Edelman, B., Moore, T., 2015. Bitcoin: Economics, technology, and governance. Journal of Economic Perspectives, 29(2), 213-38.

Bouoiyour, J., Selmi, R., 2015. What does Bitcoin look like? Annals of Economics and Finance, 16(2), 449-492.

Bouri, E., Molnár, P., Azzi, G., Roubaud, D., Hagfors, L.I., 2017a. On the hedge and safe haven properties of Bitcoin: Is it really more than a diversifier? Finance Research Letters, 20, 192-198.

Bouri E., Gupta R., Tiwari A., Roubaud, D., 2017b. Does Bitcoin Hedge Global Uncertainty? Evidence from Wavelet-Based Quantile-in-Quantile Regressions. Finance Research Letters, 23, 87-95.

Bouri E., Shahzad S. J. H., Roubaud, D. 2018. Co-explosivity in the cryptocurrency market. Finance Research Letters, https://doi.org/10.1016/j.frl.2018.07.005 
Brière, M., Oosterlinck, K., Szafarz, A., 2015. Virtual currency, tangible return: Portfolio diversification with bitcoin. Journal of Asset Management, 16(6), 365-373.

Cheah, E. T., Fry, J., 2015. Speculative bubbles in Bitcoin markets? An empirical investigation into the fundamental value of Bitcoin. Economics Letters, 130, 32-36.

Ciaian, P., Rajcaniova, M., Kancs, D. A., 2016. The economics of Bitcoin price formation, Applied Economics, 48(19), 1799-1815.

Colacito, R., Engle, R. F., Ghysels, E., 2011. A component model for dynamic correlations. Journal of Econometrics, 164(1), 45-59.

Conrad, C., Loch, K., Rittler, D., 2014. On the macroeconomic determinants of long-term volatilities and correlations in U.S. stock and crude oil markets. Journal of Empirical Finance, 29, 26-40.

Corbet, S., Lucey, B., Yarovaya, L., 2018a. Datestamping the Bitcoin and Ethereum bubbles. Finance Research Letters, 26, 81-88.

Corbet, S., Meegan, A., Larkin, C., Lucey, B., Yarovaya, L., 2018b. Exploring the dynamic relationships between cryptocurrencies and other financial assets. Economics Letters, 165, 28-34.

Demir, E., Gozgor, G., Lau, C.K.M., Vigne, S.A., 2018. Does economic policy uncertainty predict the Bitcoin returns? An empirical investigation. Finance Research Letters. https://doi.org/10.1016/j.frl.2018.01.005

Dyhrberg, A.H., 2016. Hedging capabilities of bitcoin. Is it the virtual gold? Finance Research Letters, 16, 139-144.

Engle, R. F., Ghysels, E., Sohn, B., 2013. Stock market volatility and macroeconomic fundamentals. Review of Economics and Statistics, 95(3), 776-797.

European Central Bank, 2012. Virtual Currency Schemes. October, 1-55.

Gandal, N., Hamrick, J.T., Moore, T., Oberman, T., 2018. Price manipulation in the Bitcoin ecosystem. Journal of Monetary Economics, 95, 86-96.

Guesmi, K., Saadi, S., Abid, I., Ftiti, Z., 2018. Portfolio diversification with virtual currency: Evidence from bitcoin. International Review of Financial Analysis. https://doi.org/10.1016/j.irfa.2018.03.004

Hayes, A.S., 2017. Cryptocurrency value formation: An empirical study leading to a cost of production model for valuing bitcoin. Telematics and Informatics, 34(7), 1308-1321. 
Ioannidis, C., Ka, K., 2018. Economic Policy Uncertainty and Bond Risk Premia. Available

https://editorialexpress.com/cgi-bin/conference/download.cgi?db_name=IAAE2018\& paper_id=187

Ji, Q., Bouri, E., Gupta, R., Roubaud, D. 2018. Network Causality Structures among Bitcoin and other Financial Assets: A Directed Acyclic Graph Approach. The Quarterly Review of Economics and Finance, https://doi.org/10.1016/j.qref.2018.05.016

Klein, T., Pham Thu, H., Walther, T., 2018. Bitcoin is not the New Gold - A Comparison of Volatility, Correlation, and Portfolio Performance. International Review of Financial Analysis, 59, 105-116.

Kristoufek, L., 2015. What are the main drivers of the Bitcoin price? Evidence from wavelet coherence analysis. PloS one, 10(4), art. e0123923.

Li, X., Wang, C.A., 2017. The technology and economic determinants of cryptocurrency exchange rates: The case of Bitcoin. Decision Support Systems, 95, 49-60.

Luther, W.J., Salter, A.W., 2017. Bitcoin and the bailout. The Quarterly Review of Economics and Finance, 66, 50-56.

Moser, M., Bohme, R., Breuker, D., 2013, September. An inquiry into money laundering tools in the Bitcoin ecosystem. In eCrime Researchers Summit (eCRS), 2013 (pp. 1-14). IEEE.

Ober, M., Katzenbeisser, S., Hamacher, K. 2013. Structure and anonymity of the bitcoin transaction graph. Future internet, 5(2), 237-250.

Polasik, M., Piotrowska, A. Wisniewski, T. P., Kotkowski, R., Lightfoot, G., 2015. Price fluctuations and the use of Bitcoin: An empirical inquiry. International Journal of Electronic Commerce, 20(1), 9-49.

Shahzad, S. J. H., Raza, N., Balcilar, M., Ali, S., Shahbaz, M., 2017. Can economic policy uncertainty and investors sentiment predict commodities returns and volatility?. Resources Policy, 53, 208-218.

Vasek, M., Moore, T., 2015, January. There’s no free lunch, even using Bitcoin: Tracking the popularity and profits of virtual currency scams. In International conference on financial cryptography and data security (pp. 44-61). Springer, Berlin, Heidelberg.

Yelowitz, A., Wilson, M. 2015. Characteristics of Bitcoin users: an analysis of Google 
search data. Applied Economics Letters, 22(13), 1030-1036.

Yin, L., Han, L., 2014. Macroeconomic uncertainty: does it matter for commodity prices? Applied Economics Letters, 21(10), 711-716.

\section{APPENDIX}

Table A1. Summary Statistics

\begin{tabular}{|l|r|r|r|r|r|}
\cline { 2 - 6 } \multicolumn{1}{c|}{} & \multicolumn{5}{c|}{ Variable } \\
\hline Statistic & \multicolumn{1}{c|}{ Bitcoin } & \multicolumn{1}{c|}{ MSCI } & S\&P GSCI & \multicolumn{1}{l|}{ PIMCO } & \multicolumn{1}{c|}{ GEPU } \\
\hline Mean & 0.6528 & 0.0309 & -0.0261 & 0.0028 & 146.8442 \\
\hline Median & 0.2461 & 0.0551 & 0.0000 & 0.0086 & 136.6802 \\
\hline Maximum & 49.9663 & 4.1122 & 5.4812 & 1.1228 & 307.6978 \\
\hline Minimum & -44.3784 & -5.2562 & -6.7434 & -3.9047 & 76.04353 \\
\hline Std. Dev. & 6.4187 & 0.8062 & 1.1818 & 0.3108 & 48.25016 \\
\hline Skewness & 0.2140 & -0.6018 & -0.1985 & -1.3137 & 1.16546 \\
\hline Kurtosis & 11.6750 & 8.2651 & 5.6624 & 16.9799 & 4.273017 \\
\hline Jarque-Bera & 5965.9420 & 2306.8630 & 573.0352 & 16001.7600 & 25.86379 \\
\hline$p$-value & 0.0000 & 0.0000 & 0.0000 & 0.0000 & 0.0000 \\
\hline Observations & 1898 & 1898 & 1898 & 1898 & 88 \\
\hline
\end{tabular}

Notes: Std. Dev: stands for standard deviation; $p$-value corresponds to the Jarque-Bera test with the null of normality. 